\title{
LA MISE EN ÉCONOMIE DE LA NATURE, CONTREPOINTS HISTORIQUES ET CONTEMPORAINS
}

\author{
Harold Levrel et Antoine Missemer
}

Presses de Sciences Po | «Revue économique»

2019/1 Vol. 70 | pages 97 à 122

ISSN 0035-2764

ISBN 9782724635959

Article disponible en ligne à l'adresse :

https://www.cairn.info/revue-economique-2019-1-page-97.htm

Distribution électronique Cairn.info pour Presses de Sciences Po.

(C) Presses de Sciences Po. Tous droits réservés pour tous pays.

La reproduction ou représentation de cet article, notamment par photocopie, n'est autorisée que dans les limites des conditions générales d'utilisation du site ou, le cas échéant, des conditions générales de la licence souscrite par votre établissement. Toute autre reproduction ou représentation, en tout ou partie, sous quelque forme et de quelque manière que ce soit, est interdite sauf accord préalable et écrit de l'éditeur, en dehors des cas prévus par la législation en vigueur en France. Il est précisé que son stockage dans une base de données est également interdit. 


\title{
La mise en économie de la nature, contrepoints historiques et contemporains
}

\author{
Harold Levrel* \\ Antoine Missemer**
}

\begin{abstract}
L'économie est régulièrement présentée comme vecteur d'aliénation de la nature, alors vue comme un ensemble de biens, de services et de capitaux à préserver, voire à faire fructifier, sans égard pour ses dimensions extra-économiques et symboliques. Cette mise en économie - ou économicisation - de la nature s'exprimerait à travers divers mécanismes tels que la monétarisation, la privatisation, etc. De tels phénomènes existent bel et bien. Ils méritent néanmoins d'être examinés de près pour constater, d'une part, que la littérature n'est pas encore stabilisée sur ces sujets et, d'autre part, que ces phénomènes de mise en économie ne sont ni nouveaux, ni irréversibles, ni univoques. Nous observons d'ailleurs dans la période récente des logiques d'hybridation où l'écologie investit aussi le champ économique.
\end{abstract}

\section{THE ECONOMIZATION OF NATURE, HISTORICAL AND CONTEMPORARY CONTRASTS}

Economics and economic activities are often considered as a source of alienation of nature, seen as a commodity delivering ecosystem services to be valued in order to provide benefits to people, without any considerations regarding idiosyncratic values. This economization process is supposed to occur through various mechanisms such as monetary valuation, privatization, etc. These phenomena do exist, but in-depth analysis reveals that these mechanisms are not necessarily well defined, and that these commodification processes are not new, neither irreversible, nor a one way trend. It seems that economization processes are more hybrid than assumed, and that many trends can be considered as a naturalisation of the economy and of economics.

Mots clés : économicisation, évaluation économique, services écosystémiques, économie écologique

Keywords : economization, monetary valuation, ecosystem services, ecological economics

Classification JEL : B1, B2, N5, Q2, Q5.

* CIRED, AgroParisTech, CIRAD, CNRS, EHESS, École des Ponts ParisTech, Université de Paris-Saclay. Correspondance : Centre international de recherche sur l'environnement et le développement, Jardin tropical, 45 bis avenue de la Belle Gabrielle, 94736 Nogent-sur-Marne Cedex, France. Courriel : harold.levrel@agroparistech.fr

** CNRS, CIRED (UMR 8568). Correspondance : Centre international de recherche sur l'environnement et le développement, Jardin tropical, 45 bis avenue de la Belle Gabrielle, 94736 Nogentsur-Marne Cedex, France. Courriel : missemer@centre-cired.fr (auteur correspondant)

Nous tenons à remercier Thierry Brunelle, Jean-Christophe Bureau, Romain Felli, Fabrice Flipo, Louis-Gaëtan Giraudet, Alain Karsenty, Yann Laurans, Alain Nadaï, Antonin Pottier, Rémi Prudhomme, Fabien Quétier, François Salanié et Bruno Villalba pour leurs orientations et soutien dans ce projet. Merci également aux participants du projet PSL « Humanités environnementales ». Merci enfin aux rapporteurs et aux éditeurs de la Revue économique pour leurs précieuses indications qui nous ont permis d'améliorer substantiellement ce texte. Nous restons seuls responsables des lignes interprétatives et des potentielles maladresses qui suivent. 


\section{INTRODUCTION}

Que les dynamiques de destruction de la nature aient pour origine des forces économiques, en particulier dans le cadre du système capitaliste industriel tel qu'il s'est développé à partir du XIX ${ }^{\mathrm{e}}$ siècle, la plupart des observateurs en conviennent. Que ces mêmes forces économiques puissent aujourd'hui fournir des outils de protection de la nature fait en revanche débat. La question de la mise en économie - ou économicisation - de l'environnement est aujourd'hui traitée au sein de différents champs disciplinaires : l'écologie (Walker et al. [2009]), la science politique (Felli [2015], [2016]), l'histoire des techniques (Bonneuil [2015]; Pestre [2016]), l'anthropologie (Fairhead, Leach et Scoones [2012]; Greene [2004]), l'économie écologique (Boisvert [2016]; Gómez-Baggethun et al. [2010]; Gómez-Baggethun et Ruiz-Pérez [2011]; Kosoy et Corbera [2010]; Levrel [2013]; Spash [2011]) et la géographie critique (Castree [2003], [2006], [2010]; Castree et Henderson [2014]; Liverman [2004]; McAfee [2010]; McAfee et Shapiro [2010]; Robertson [2004], [2006], [2012]; Sullivan [2013]). Le message général véhiculé par ces différents travaux consiste en une critique des phénomènes de mise en économie de la nature, accusés de biaiser le regard porté par les communautés humaines sur leur environnement. Vue comme un ensemble de capitaux (naturels) et de services (écosystémiques), la nature ne devient qu'un instrument de production sans dimension extra-économique ou symbolique, avec in fine des effets néfastes et contreproductifs sur la sauvegarde des ressources et des milieux.

Dans cet article, nous souhaitons analyser et discuter les processus habituellement associés à la mise en économie de la nature (monétarisation, privatisation, marchandisation, commodification, financiarisation), en soulignant certaines limites aux démonstrations proposées dans la littérature. L'enjeu de notre contribution n'est pas de dire que les phénomènes de mise en économie de la nature n'existent pas. Il n'est pas non plus de porter un jugement normatif sur l'efficacité ou le bien-fondé de cette mise en économie. Notre objectif est en revanche de clarifier ce que signifie la mise en économie de la nature, et d'évaluer, à travers différents contrepoints historiques et contemporains, son caractère nouveau, univoque et irréversible.

Pour construire notre analyse, une première partie s'appuie sur une revue de la littérature pour préciser ce que signifient les concepts de monétarisation, privatisation, marchandisation, commodification et financiarisation de la nature tels qu'ils apparaissent habituellement dans les analyses. Selon nous, à la lecture de cette littérature, il semble possible de comprendre la mise en économie de la nature comme une chaîne causale reliant ces différents mécanismes entre eux. La deuxième partie de l'article s'attache à souligner l'ancienneté des phénomènes de mise en économie de la nature. Nous nous attardons en particulier sur deux moments historiques : les $\mathrm{XVII}^{\mathrm{e}}-\mathrm{XVIII}^{\mathrm{e}}$ siècles et le tournant du $\mathrm{XX}^{\mathrm{e}}$ siècle, pour mettre en exergue l'existence à ces époques-là de phénomènes très proches dans leurs caractéristiques de ceux que nous rencontrons aujourd'hui. Enfin, une troisième partie en vient à la période contemporaine et à l'évaluation de certains modes de régulation qui se développent actuellement, pour voir qu'ils ne correspondent pas à proprement parler à une mise en économie de la nature. Cette contribution s'achève par quelques remarques de conclusion. 


\section{MATÉRIAU, MÉTHODE ET DÉFINITIONS : UNE REVUE DE LA LITTÉRATURE}

Pour mener à bien notre discussion des processus de mise en économie de la nature, nous avons cherché à comprendre comment les mécanismes qui y sont associés (monétarisation, marchandisation, etc.) ont été jusque-là définis et commentés dans la littérature, aussi bien en économie que dans d'autres disciplines. Une première étape a consisté à réaliser une analyse bibliographique à partir de la plate-forme Web of Science ${ }^{1}$. Nous avons croisé le mot clé «nature » avec plusieurs concepts ${ }^{2}$ : " monetization ", "privatization ", " commodification », « commoditization ", " financialization », « capitalization 》, « marketization $»^{3}$. Neuf cent quatre-vingts articles ont ainsi été recensés. Pour identifier les enjeux clés traités par ces textes, nous nous sommes focalisés sur les travaux académiques les plus commentés, en nous intéressant aux quinze contributions les plus citées. Il est apparu rapidement que le mot «nature » n'était pas assez discriminant ${ }^{4}$. Nous avons donc substitué «nature » par «biodiversity " (plus restrictif mais tout aussi adapté à l'étude des processus de mise en économie dans la littérature récente). L'analyse bibliographique a permis d'identifier 139 articles publiés entre 1998 et 2016 (figure 1). Sur les quinze références les plus citées, quatorze nous sont apparues en relation avec la question de la mise en économie de la nature. Nous nous sommes donc appuyés sur ces résultats pour donner une photographie la plus fidèle possible de l'état de la littérature. En complément, nous avons également mobilisé des travaux, en particulier francophones, qui n'apparaissaient pas nécessairement dans les résultats de nos requêtes, mais qui nous permettaient d'approfondir certaines questions entourant les phénomènes de mise en économie de la nature. L'ensemble de ces sources est répertorié en bibliographie.

L'analyse menée sur Web of Science nous a permis de mesurer l'essor récent des débats sur la mise en économie de la nature. Non seulement le nombre d'articles publiés sur cette thématique croît depuis le début des années 2010 (figure 1), mais il semble que ces articles soient de plus en plus commentés et cités (figure 2).

Parmi les différents mécanismes associés à la mise en économie de la nature (monétarisation, marchandisation, privatisation, etc.), tous n'ont pas la même importance dans la littérature. Les notions de commodification et de privatisation sont clairement les deux concepts qui apparaissent le plus souvent. Les autres concepts sont bien moins mentionnés (tableau 1). Ces différentes notions ne sont par ailleurs pas toujours définies clairement, même si nous avons cherché à cerner quelques définitions de référence parmi les travaux les plus cités. En reprenant les quinze premiers articles issus de nos analyses bibliographiques pour la mise en économie de la nature ("nature ») et de la biodiversité (" biodiversity»), nous avons pu identifier vingt-trois contributions ${ }^{5}$ dans lesquelles il est possible de faire ressortir quelques éléments de définition ( $c f$. tableau 1 et tableaux A1 et A2

1. http://apps.webofknowledge.com/.

2. Y compris leurs variantes linguistiques et orthographiques.

3. La notion française de marchandisation, dans son sens précis de transaction marchande, a pour équivalents anglais commoditization et marketization (Castree [2010]; McAfee et Shapiro [2010]).

4. Pour obtenir les quinze références les plus citées, il nous a fallu aller jusqu'à la vingt-sixième référence, onze références n'étant pas pertinentes pour notre sujet.

5. Vingt-trois car sept articles sont communs aux deux analyses bibliographiques. 
Figure 1. Nombre et dates des publications associant « biodiversity » et mise en économie de la nature (via différents concepts)

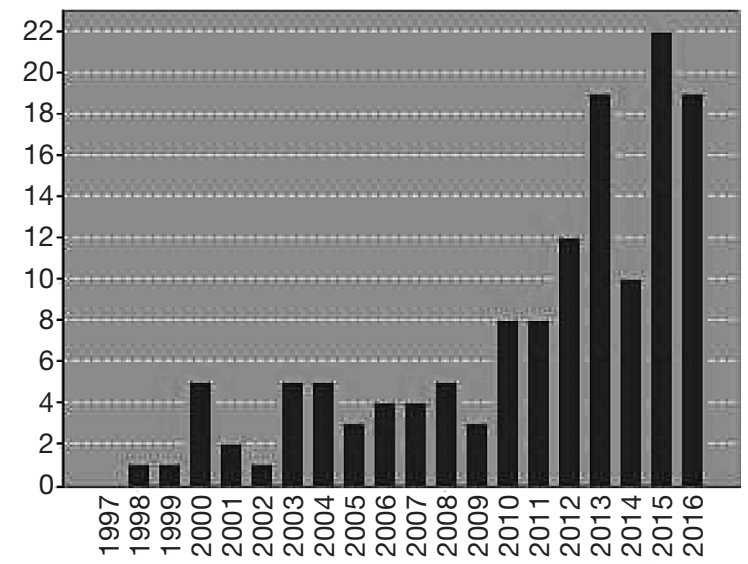

Source: Web of Science, mars 2017.

Figure 2. Nombre et dates des citations des articles associant " biodiversity » et mise en économie de la nature (via différents concepts)

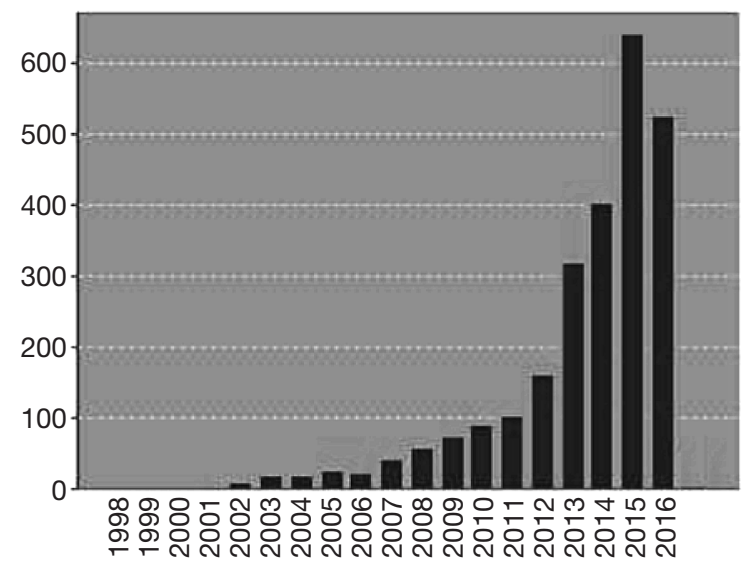

Source: Web of Science, mars 2017.

en annexe). Dix-huit articles mobilisent la notion de commodification, mais seuls quatre en fournissent une définition. Dix-sept font référence à la privatisation, dont sept en précisent le sens. La capitalisation et la marketization ne sont jamais définies, alors que la financiarisation est définie deux fois sur cinq. Pour la monétarisation, nous n'avons pu repérer qu'une seule définition dans notre échantillon. Enfin, deux des trois articles mobilisant la notion de commoditization (plus étroite que commodification) en fournissent un sens précis. Bien que notre échantillon soit réduit, il fait état des contributions les plus commentées et citées, et nous semble à cet égard suffisamment représentatif. Il apparaît donc qu'un certain flou sémantique demeure dans l'usage des termes associés à la mise en économie de la nature, en dépit des quelques définitions que nous avons pu repérer (tableau 1). 
Tableau 1. Définitions des concepts associés à la mise en économie de la nature

\begin{tabular}{|c|c|c|}
\hline Concepts & $\begin{array}{l}N^{\text {bre }} \text { d'articles } \\
\quad(n=139)\end{array}$ & $\begin{array}{l}\text { Définition utilisée dans les principaux articles } \\
\qquad(\mathrm{n}=23)\end{array}$ \\
\hline Commodification & 72 & $\begin{array}{l}\text { Expansion of market trade to previously non-marketed } \\
\text { areas (Gómez-Baggenthun et Ruiz-Pérez [2011]) }\end{array}$ \\
\hline Privatization & 48 & $\begin{array}{l}\text { Assignation of legal title to a named individual, group or } \\
\text { institution (Castree [2003]) }\end{array}$ \\
\hline Capitalization & 13 & - \\
\hline Monetization & 11 & $\begin{array}{l}\text { Exchange values through pricing (Gómez-Baggenthun } \\
\text { et Ruiz-Pérez [2011]) }\end{array}$ \\
\hline Financialization & 4 & $\begin{array}{l}\text { Drawing into financial circulation aspects of life that } \\
\text { previously lay outside it (Fairhead, Leach et Scoones } \\
\text { [2012]) }\end{array}$ \\
\hline Marketization & 4 & $\begin{array}{l}\text { Rendering alienable and exchangeable things that might } \\
\text { not previously have been subject to a market logic } \\
\text { expressed through (Castree [2010]) }\end{array}$ \\
\hline Commoditization & 3 & Genuine market transactions (McAfee et Shapiro [2010]) \\
\hline
\end{tabular}

La précision des concepts semble dépendre de l'objet étudié. Parmi les vingttrois articles les plus cités, les termes liés à la mise en économie de la nature sont mieux définis dans le domaine de l'eau que dans celui de la biodiversité (voir notamment Budds et McGranahan [2003] et Bakker [2007]). Cela est peut-être dû à davantage d'ancienneté dans les études sur l'eau, et donc à une littérature conceptuellement plus consolidée.

Plus globalement, d'une littérature à l'autre, la mise en économie de la nature n'est pas définie à partir des mêmes mécanismes. Certains travaux s'attachent à l'analyse d'un type de phénomène en particulier (par exemple Boisvert, Caron et Rodary [2004] pour la privatisation, Sullivan [2013] pour la financiarisation, Büscher [2010] ou encore Duffy [2014] pour la commodification, etc.). D'autres construisent une critique plus générale (Castree et Henderson [2014]; O’Neill [2007]; Pestre [2016]). Tout en conservant une vision d'ensemble, certains textes s'attachent à traiter distinctement les différents mécanismes à l'œuvre, sans amalgame (Castree [2003]; Bakker [2007]; Budds et McGranahan [2003]; Gómez-Baggethun et Ruiz-Pérez [2011]). D'autres insistent sur les enchaînements causaux entre mécanismes (monétarisation, privatisation, marchandisation, etc.), au risque de ne pas toujours définir clairement la façon dont ces enchaînements s'opèrent (Banerjee [2003]; Dauguet [2015]; Fairhead, Leach et Scoones [2012]; Gómez-Baggethun et al. [2010]; Luck et al. [2012]; O'Neill [2007]; Robertson [2004], [2006], [2012]; Sullivan [2013]). Ces articulations entre mécanismes associés à la mise en économie de la nature nous semblent au cœur de la littérature. Nous pourrions ainsi reconstruire une chaîne causale : la mise en économie commencerait par l'usage d'un lexique économique pour parler des éléments de la nature; ce lexique déboucherait sur une vision instrumentale (et utilitariste) de la nature; s'opérerait alors une mise en équivalence (souvent monétaire) de ses composantes; cette mise en équivalence faciliterait la privatisation, qui déboucherait sur la marchandisation de la nature; et le stade 
le plus avancé de cette marchandisation serait celui de la financiarisation ${ }^{6}$. Cette chaîne causale, reproduite sur la figure 3 , a les avantages et les inconvénients de la synthèse. Elle donne à voir ce que signifie la mise en économie de la nature, mais nul doute que certains travaux ne s'y retrouveraient pas totalement. Il nous semble néanmoins qu'elle offre une photographie assez fidèle de ce que la littérature entend, le plus souvent, par mise en économie de la nature, notamment lorsque le champ sémantique reste imparfaitement défini. Nous aurons l'occasion dans les sections qui suivent de préciser et d'évaluer l'importance de chaque chaînon, ainsi que les enchaînements causaux censés les relier.

Figure 3. Chaîne causale de mise en économie de la nature

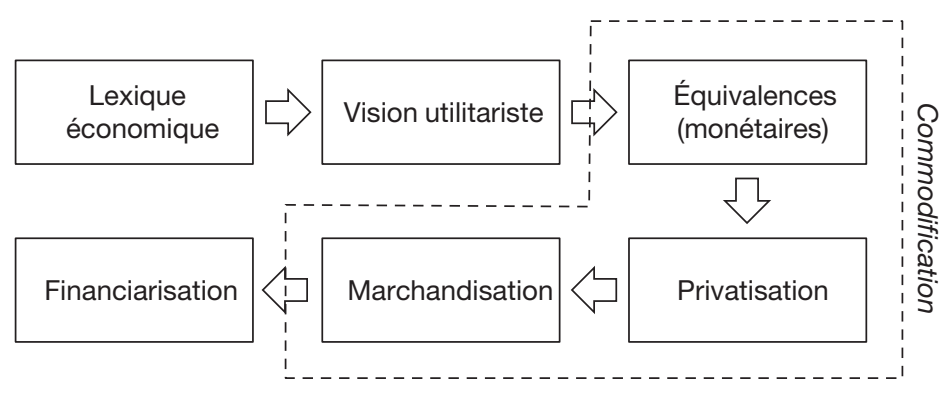

Pour résumer, la mise en économie de la nature (et de la biodiversité), telle qu'elle est définie dans la littérature, regroupe une diversité de mécanismes qui restent souvent imparfaitement définis. La diversité des objets d'étude et des études de cas conduit à des discussions toujours plus nombreuses de ces sujets, en particulier depuis le début des années 2010. En repérant quelques définitions clés au sein des travaux les plus cités, et en remarquant que la mise en économie est souvent présentée comme une succession de processus, tantôt indépendants, tantôt consubstantiels, il semble possible de résumer tous ces enjeux à travers un schéma causal simple (figure 3) qui met en évidence à la fois la diversité des processus en jeu (lexique, monétarisation, privatisation, marchandisation, etc.) et les potentielles relations entre ces processus.

Définie ainsi, la mise en économie de la nature est régulièrement présentée comme un phénomène récent, amplifié de façon décisive par l'accession du néolibéralisme aux responsabilités politiques dans les années 1980 (Castree [2008a], [2008b]; Gómez-Baggethun et al. [2010]; Robertson [2004]; Spash [2011]). Surtout, ce caractère nouveau se couplerait d'une dimension univoque et irréversible des processus : dès lors que la chaîne causale de mise en économie se déclencherait, il serait difficile d'en réchapper. Sur ces différents points, une analyse précise mérite d'être menée pour offrir une vision nuancée des processus de mise en économie de la nature.

6. La notion de commodification, davantage présente dans la littérature anglo-saxonne, recouvre à la fois des processus de mise en équivalence, de privatisation et de marchandisation. Sur cette notion que nous n'aborderons dans la suite que de façon périphérique, voir notamment Castree [2003] et Espeland et Stevens [1998]. 


\section{CONTREPOINTS HISTORIQUES SUR LA MISE EN ÉCONOMIE DE LA NATURE}

\section{Quatre siècles d'échanges lexicaux et conceptuels}

À première vue, l'usage abondant d'un lexique économique pour parler des milieux naturels est une réalité récente (Åkerman [2003]; Castree et Henderson [2014], p. 24; Nadal [2016]). L'exemple de la notion de service écosystémique, définie dans les années 1970 et 1980 (Boisvert [2015b]; Ehrlich et Mooney [1983]; Gómez-Baggethun et al. [2010]), est à cet égard illustratif. En focalisant l'attention sur les bénéfices offerts par la nature à l'homme, cette notion inscrit la protection, la destruction ou la restauration de l'environnement dans des logiques de justifications fondées sur les calculs coûts-bénéfices. Les stocks de ressources exploités ne sont d'ailleurs pas les seuls éléments considérés; toutes les interactions écologiques qui offrent, directement ou indirectement, des bénéfices à l'homme (comme la filtration de l'eau), ainsi que toutes les composantes de la biodiversité qui fournissent des bénéfices non marchands (comme les activités récréatives de la nature), sont ainsi désignées. Mais si la notion de service écosystémique est bel et bien récente, l'usage plus général d'un lexique économique pour décrire la nature et ses dynamiques est loin d'être nouveau.

Les échanges lexicaux et conceptuels entre économie et sciences naturelles sont en fait anciens et datent, au moins, du XVII ${ }^{e}$ siècle. Charlton [1658] définit à l'époque ce qu'il appelle une «économie animale » pour décrire les mécanismes se rapportant aux fonctions internes d'un organisme et aux interactions de cet organisme avec son environnement (Balan [1975]). Peu après, à l'époque des Lumières, naît une «économie rustique », définie par l'Encyclopédie de Diderot et d'Alembert ([1755], p. 349) comme « l'art de connaitre tous les objets utiles et lucratifs de la campagne, de se les procurer, de les conserver, et d'en tirer le plus grand avantage possible. Cette manière de s'enrichir est d'une étendue prodigieuse : c'est un tribut imposé sur tous les êtres de la nature; les éléments mêmes n'en sont pas exceptés ». Au XIX siècle, Haeckel ([1874], p. 637-638) donne naissance à l'écologie en la définissant comme « une économie de la nature ». Et depuis, les écologues hésitent peu à utiliser des concepts empruntés à l'économie tels que les notions de productivité, de consommateur, de producteur, de richesse, etc. Dans ses travaux sur les rapports entre savoir économique et philosophie naturelle, Schabas [2005] a abondamment mis en exergue ces proximités non seulement lexicales, mais aussi conceptuelles, en soulignant une intrication forte entre sciences naturelles et savoirs sur le monde social. La réciprocité des échanges témoigne d'ailleurs de cette intrication. Dès le XVIII ${ }^{\mathrm{e}}$ siècle, certains écrits de nature économique s'inspirent des dynamiques observables dans la nature. Quesnay [1758], médecin et économiste, décrit la circulation des richesses dans l'économie comme la circulation des fluides dans un organisme. Au tournant du XIX ${ }^{e}$ siècle, ce sont les économistes classiques qui mobilisent les lois de la nature (gravitation, circulation des fluides) pour construire leurs édifices théoriques (voir Schabas [2005], chap. V et VI). Plus tard, Podolinsky [1880a], [1880b] propose une description de l'économie à partir des relations existant entre le règne végétal (créateur de richesse) et le règne animal (destructeur de richesse), en formalisant les dynamiques humaines d'accumulation et de dispersion des richesses, évaluées en unités d'énergie. Plus récemment, les théories 
évolutionnaires de la firme (Nelson et Winter [1982]) ne sont pas étrangères à l'incorporation de dynamiques biologiques dans les raisonnements économiques.

En réalité, depuis quatre siècles au moins, ces échanges lexicaux et conceptuels révèlent une philosophie et une éthique anthropocentrées qui font figure de permanence historique en Occident, aussi bien en sciences naturelles qu'en économie (Wolloch [2016]). Le siècle des Lumières a sécularisé la cosmologie de la Genèse dans laquelle la nature est au service de l'homme, et où l'utile préside à toute logique d'action sur l'environnement. Il n'est donc pas surprenant que les sciences naturelles, prises dans cette philosophie anthropocentrée, aient régulièrement mobilisé un vocabulaire issu de l'économie, définie comme le savoir sur l'usage des choses. Les premiers naturalistes (Linné, Buffon) défendaient ainsi une vision très instrumentale de la nature, ce qui a pesé sur leurs propositions scientifiques et sur leurs usages conceptuels (Roger [1993]).

Cette histoire longue apporte une première nuance au caractère nouveau des phénomènes de mise en économie de la nature. Bien entendu, la dimension anthropocentrée des rapports homme-nature en Occident est bien connue, et nombreux sont les commentateurs à admettre que, de ce point de vue, la mise en économie de la nature n'est pas nouvelle (Bonneuil [2015]; Boudia [2016]; Fairhead, Leach et Scoones [2012]; Gómez-Baggenthun et al. [2010]; Pestre [2016]; Robertson [2012]). Ces mêmes commentateurs soulignent toutefois quelques caractéristiques singulières qui tendraient à rendre la période contemporaine inédite : la mise en économie serait aujourd'hui totale, des gènes aux écosystèmes globaux; elle considérerait la nature non plus comme une marchandise mais comme un actif générant des services écosystémiques échangeables sur des marchés; et les acteurs privés deviendraient les pivots des régulations. Il nous semble toutefois que plusieurs de ces caractéristiques ont aussi pu exister par le passé. Nous proposons de nous arrêter sur deux moments, d'abord les XVII ${ }^{\mathrm{e}}$ et $\mathrm{XVIII}^{\mathrm{e}}$ siècles puis le tournant du $\mathrm{XX}^{\mathrm{e}}$ siècle, pour montrer que ces apparentes spécificités contemporaines trouvent des échos historiques.

\section{La mise en économie des forêts, de la faune et des zones humides auX XVII ${ }^{e}$ et $\mathrm{XVIII}{ }^{e}$ siècles}

Plusieurs épisodes historiques nous permettent de mettre en perspective les phénomènes de mise en économie de la nature. En particulier, les $\mathrm{XVII}^{\mathrm{e}}$ et $\mathrm{XVIII}^{\mathrm{e}}$ siècles offrent un regard singulier sur les processus de privatisation et de marchandisation, voire de financiarisation. À ce moment-là, en Grande-Bretagne comme en France, la question de la propriété des espaces naturels, de la faune et de la flore, a subi d'importantes évolutions.

Dans sa célèbre analyse sur les origines du Black Act, législation britannique de 1723 contre le braconnage et le pillage de ressources forestières, Thompson [1975] met en évidence les mécanismes légaux qui ont conduit à privatiser d'importantes parcelles de forêts, en particulier dans la forêt de Windsor et dans le Hampshire. Ces enclosures, initiées à la fin du XVII ${ }^{\mathrm{e}}$ siècle et souvent permises par des privilèges royaux concédés à des notables proches de la Couronne, ont mis à mal des pratiques coutumières où demeuraient des droits d'usage, souvent tacites, sur les espaces et les ressources. Comme Thompson l'indique ([1975], p. 241), cette privatisation de la forêt et de ses ressources s'est inscrite dans un climat 
général de renforcement de la propriété privée. À l'égard de notre sujet, force est de constater que ces pratiques ne sont pas sans rappeler les processus d'accaparement des terres (land-grabbing) mis en évidence aujourd'hui dans la littérature (Borras Jr. et al. [2011]; Fairhead, Leach et Scoones [2012]). L'accaparement des terres consiste dans la privatisation de grands espaces agricoles ou forestiers au profit d'acteurs souvent extérieurs (entreprises, ONG, etc.). Les finalités de ces privatisations (prétendue conservation des milieux, sécurisation des approvisionnements alimentaires, exploitation durable des ressources) ne sont pas forcément les mêmes qu'au XVIII ${ }^{\mathrm{e}}$ siècle, mais trois caractéristiques semblent néanmoins communes : la présence d'acteurs privés argentés, la complicité des autorités (locales ou nationales) et la remise en cause de pratiques coutumières de gestion des espaces et des ressources. Bien que le contexte soit différent, la privatisation contemporaine de la nature semble avoir, à cet égard, des précédents historiques.

La question des droits de chasse en France, toujours au XVIII ${ }^{\mathrm{e}}$ siècle, interroge également le caractère nouveau des mécanismes de mise en économie de la nature. Avant la Révolution française, les droits de chasse étaient une prérogative de classe. Au sommet se trouvaient les droits dont disposait le roi, puis ceux des nobles en fonction de leur importance et enfin ceux des bourgeois et du clergé. La reconnaissance de droits privés sur la faune à travers les droits de chasse apparaît à la suite de la Révolution française avec la loi du 22 frimaire an 7 (Estève [2004]). Le droit de chasse est accolé au droit de propriété foncier et correspond à un droit privé sur la faune très important, puisqu'il en autorise la destruction (Domas-Descos [2012]). À l'égard des problématiques contemporaines, il est intéressant de noter qu'à partir de la Révolution, avec les droits de chasse, le droit d'usage sur la faune devient un droit autonome du droit d'usage de la terre ${ }^{7}$. Le propriétaire foncier peut louer en parallèle un droit de fermage et un droit de chasse, ce dernier étant négociable sur un marché de droits (Estève [2004]). La loi du 26 juin 1824 mentionne un prix de location pour la chasse pouvant aller jusqu'à $4 \%$ de la valeur du terrain. Le développement de ce marché est tel qu' " au début de la $\mathrm{III}^{\mathrm{e}}$ République le marché du bois de chasse était en quelque sorte saturé. Toutes les belles forêts avaient été plus ou moins louées » (Estève [2004], p. 81). L'exemple des droits de chasse indique qu'il existait déjà au XVIII ${ }^{e}$ siècle des droits d'usage (et de destruction) sur la faune, considérée aujourd'hui comme une composante de la biodiversité, et surtout que ces droits d'usage n'étaient pas liés qu'à l'exploitation d'une ressource pour des besoins alimentaires. En effet, compte tenu des acteurs intervenant sur ce marché de droits (souvent d'anciens nobles ou bourgeois argentés), la chasse constituait aussi et surtout une activité récréative, ce qui suggère que les services récréatifs de certaines composantes de la biodiversité connaissaient déjà, à l'époque, des formes de privatisation et de marchandisation.

Le dernier exemple des $\mathrm{XVII}^{\mathrm{e}}-\mathrm{XVIII}{ }^{\mathrm{e}}$ siècles utile à la mise en perspective des phénomènes de mise en économie de la nature réside dans la marchandisation, voire la financiarisation, progressive des zones humides en France (Derex [2001], [2017]). Cette histoire nous révèle que la dynamique de ces espaces et leur conservation ont vite été dépendantes des usages marchands (élevage, production de sel, récolte de tourbe et de sangsues, pisciculture). La création des zones humides a été elle-même souvent motivée par des considérations économiques.

7. Remarquons que Thompson ([1975], p. 98) décrit l'existence de droits de chasse similaires en Angleterre, octroyés de façon discrétionnaire par certains officiers forestiers. 
Ainsi, dès le $\mathrm{XII}^{\mathrm{e}}$ siècle, les marchés ont été à l'origine de nombreux marais, à travers le développement d'étangs destinés à approvisionner les nobles - puis les bourgeois - en poissons frais (carpe et brochet). Ces étangs ont donné naissance à de grandes zones humides (par exemple la Brenne, la Sologne et la Dombes) dont la conservation n'a évidemment plus pour objectif d'approvisionner les populations riveraines en poissons. Ces logiques marchandes ont aussi pu conduire à détruire des zones humides, mais ce sont bien plus les politiques publiques d'assèchement, fondées sur des concessions et des avantages fiscaux offerts aux compagnies spécialisées, qui ont généré ces destructions (Derex [2001]). Les instruments fiscaux et réglementaires mis en place à l'époque ont conduit à des comportements de spéculation par des compagnies d'assèchement prenant les traits de « véritables entreprises capitalistes imposant une nouvelle logique sur [les] espaces et sur les populations » (ibid., p. 30). Ces logiques spéculatives sur des espaces naturels ne sont pas sans rappeler certains aspects de la financiarisation de la nature dénoncée aujourd'hui (Büscher [2010]; Sullivan [2013]). Ces politiques incitatives ont commencé au XVII ${ }^{\mathrm{e}}$ siècle en France pour ne véritablement s'arrêter qu'au début des années 2000 avec l'adoption de la Directive-cadre sur l'eau (2000).

À maints égards, les XVII ${ }^{\mathrm{e}}$ et $\mathrm{XVIII}^{\mathrm{e}}$ siècles offrent plusieurs exemples de privatisation et de marchandisation, voire de financiarisation, de la nature dont les caractéristiques sont proches des réalités contemporaines. L'intervention d'acteurs privés dans la définition et la jouissance des droits de propriété, la mise en place de marchés, en particulier de droits, l'émergence de logiques spéculatives sur les espaces naturels sont autant de phénomènes parfois présentés comme nouveaux mais qui trouvent des échos historiques importants. Le tournant du $\mathrm{XX}^{\mathrm{e}}$ siècle offre lui aussi des contrepoints significatifs.

\section{Services écosystémiques et régulations des usages de la nature au tournant du $\mathrm{xx}^{\mathrm{e}}$ siècle}

Dans la lignée des échanges lexicaux et conceptuels entre sciences naturelles et économie apparaît à la fin du XIX ${ }^{\mathrm{e}}$ siècle ce que l'on nomme alors « économie ornithologique », " économie zoologique », " économie botanique » ou encore « économie entomologique » (Kellogg et Doane [1915]; Palmer [1899]; Rhoads [1898]). L'économie ornithologique, en particulier, met en évidence une conception de la nature, en l'occurrence ici des oiseaux, très proche de la conception véhiculée par la notion de service écosystémique (Kronenberg [2014], p. 172; voir aussi Boisvert [2015b], [2016] et Levrel et al. [2015b]). Palmer décrit l'économie ornithologique en 1899 (p. 259) comme " the study of birds from the standpoint of dollars and cents. It deals with birds and their relation to agriculture, horticulture, trade and sports; it traits of species important to the farmer, the fruitgrower, the game dealer, the milliner, and the sportsman " (cite par Kronenberg [2014], p. 166). L'idée de monétarisation des services non marchands fournis par la nature, souvent dénoncée pour la période contemporaine (Feuillette et al. [2016]; Levrel [2013]; O'Connor et Spash [1999]), fait ici son apparition de façon flagrante. Le principal service évalué à l'époque est celui de la régulation des espèces nuisibles par des oiseaux protecteurs de cultures. En 1885, la division d'économie ornithologique du département américain de l'Agriculture souligne ainsi que 90000 dollars de subventions sont susceptibles d'éliminer 
130000 oiseaux qui auraient causé pour 1875 dollars de dommages, alors que si les oiseaux avaient été laissés en vie, ils auraient produit 3,9 millions de dollars de bénéfices (Kronenberg [2014], p. 171). Le calcul du manque à gagner est évalué à partir de ce que l'on connaît aujourd'hui sous le nom de méthode des coûts de remplacement : l'auteur évalue les dégâts réalisés par des nuisibles sur les récoltes, et considère la part de ces destructions qui aurait pu être évitée si on avait laissé les espèces d'oiseaux utiles sur les parcelles agricoles. Certes, la méthode est approximative, et les résultats sont évidemment discutables, mais l'idée de dégager des ordres de grandeur et de peser sur la décision publique est là, comme dans beaucoup d'exercices contemporains de monétarisation des services écosystémiques (Braat et Ten Brink [2008]; Chevassus-au-Louis, Salles et Pujol [2009]; Costanza et al. [1997]; Laurans et al. [2001]). L'économie ornithologique, qui offre une mise en perspective utile aux phénomènes de monétarisation de la nature, a connu un franc succès des années 1870 aux années 1930, avant de reculer face aux usages croissants d'intrants chimiques dans l'agriculture, jugés plus fiables et plus pratiques à gérer que les oiseaux.

Le tournant du $\mathrm{xx}^{\mathrm{e}}$ siècle fait figure, aux États-Unis, de moment décisif dans la mise au point de modes de régulation de la nature. En marge de l'économie ornithologique, tout un mouvement de protection de l'environnement se développe dès la fin du XIX ${ }^{e}$ siècle, d'abord à travers deux visions opposées : une vision contemplative incarnée par le préservationnisme de John Muir, où les espaces naturels doivent être sanctuarisés, avec une finalité tout autant esthétique que naturaliste (voir Bergandi et Galangau-Quérat [2008] et Hays [1959]); et une vision instrumentale portée par le conservationnisme de Gifford Pinchot, où les ressources et les espaces méritent d'être protégés afin d'être valorisés pour le bien-être humain (voir Fox [1981], Hays [1959] et Missemer [2017]). Dans les premières années du $\mathrm{xx}^{\mathrm{e}}$ siècle, c'est finalement le conservationnisme qui va l'emporter, en présidant à la gestion des espaces naturels sous l'administration Roosevelt (voir Fox [1981], p. 139-140). Comme l'économie ornithologique, les travaux conservationnistes fournissent des exemples d'évaluations monétaires de certains services, en vue de produire des analyses coûts-bénéfices autour de projets de développement ou, inversement, de conservation. Dans le rapport de la National Conservation Commission [1909] est cité le service indirect fourni par la forêt pour la perpétuation de certaines espèces de poissons et de gibier. Semblent également évoqués certains services récréatifs : "Forests not only grow timber, but they hold the soil and they conserve the streams. They abate the wind and give protection from excessive heat or cold. Woodlands make for the fiber, health, and happiness of each citizen and of the nation. The fish which live in forest waters furnish each year \$21,000,000 worth of food, and not less than half as much is furnished by the game which could not exist without the forest 》 (p. 51). Les méthodes d'évaluation monétaire employées restent relativement floues mais participent de l'expertise.

Le conservationnisme interroge un autre aspect des processus de mise en économie de la nature, à savoir l'influence de l'utilitarisme sur les pratiques de régulation. Pinchot ([1910], p. 48) ne cache pas l'ascendance utilitariste de son mouvement, dans la lignée de Bentham [1789] et de Mill [1863], en lui donnant pour devise la recherche du « plus grand bonheur du plus grand nombre, le plus longtemps possible », une maxime proche de la devise benthamienne qui a conduit certains observateurs à qualifier le conservationnisme d'utilitarisme « amendé » (McConnell [1954], p. 469). Il semble donc, d'une part, que le regard utilitariste 
dans les politiques de régulation de la nature soit ancien, et que, d'autre part, certains des enchaînements causaux décrits plus haut (figure 3) ne semblent pas résister au contre-exemple du conservationnisme. En effet, bien qu'utilitaristes et favorables aux évaluations monétaires de la nature, les conservationnistes s'opposaient à la gestion privée, et surtout aux dérives monopolistiques de la propriété privée, dans le domaine des ressources et des espaces naturels (Pinchot [1908], p. $745 ;$ [1910], p. 29-30; [1924], p. 65), certains qualifiant même la privatisation des ressources naturelles d' « illogique » et d' « absurde» (Bruce [1909], p. 160). Le lien de causalité suggéré entre utilitarisme, monétarisation et privatisation de la nature (Gómez-Baggenthun et al. [2010]) est donc susceptible de se heurter à l'exemple du conservationnisme du début du $\mathrm{Xx}^{\mathrm{e}}$ siècle - un contrepoint historique utile pour mieux cerner les enjeux de la mise en économie de la nature.

D'autres moments historiques, en sus des $\mathrm{XVII}^{\mathrm{e}}$-XVIII ${ }^{\mathrm{e}}$ siècles et du tournant du $\mathrm{Xx}^{\mathrm{e}}$ siècle, pourraient venir alimenter la réflexion sur la contemporanéité des phénomènes de mise en économie de la nature, comme les premières décennies du XIX ${ }^{\mathrm{e}}$ siècle et l'essor (temporaire) d'évaluations monétaires de certains services récréatifs ou culturels rendus par les paysages (Fressoz [2012], [2013]). L'enjeu n'est toutefois pas pour nous d'être exhaustifs, mais de simplement rappeler que chaque chaînon de la mise en économie de la nature semble connaître des précédents historiques, et que certains enchaînements causaux sont aussi susceptibles d'être nuancés. Certains attributs présentés comme spécifiques à la période contemporaine, comme l'importance des acteurs privés ou la vision d'une nature comme un ensemble d'actifs (en particulier de la faune aux écosystèmes), trouvent des échos dans le passé. Le caractère inédit de la mise en économie de la nature, même dans ses apparentes propriétés, mérite donc sans doute discussion.

\section{CONTREPOINTS CONTEMPORAINS SUR LA MISE EN ÉCONOMIE DE LA NATURE}

\section{L'économie comme un langage parmi d'autres}

La période contemporaine est celle qui fait l'objet des plus grandes attentions de la part des observateurs des phénomènes de mise en économie de la nature. $\mathrm{Au}$ sommet de la chaîne causale, la question du lexique joue un rôle important. Il est ainsi avancé que l'apparition de la notion de service écosystémique s'est faite aux dépens de celle de biodiversité (Fourcade [2011]; Lévêque [2007]). La première, entrée en concurrence avec la seconde, deviendrait hégémonique et ferait disparaître les valeurs associées à la conservation de la biodiversité, au profit de valeurs servicielles et donc strictement économiques ${ }^{8}$. Cette lecture mérite analyse. L'usage des deux notions dans le domaine scientifique suit une tendance à la hausse depuis de nombreuses années, mais sans que l'on observe d'inflexion dans l'usage de la notion de biodiversité au profit de celle de service écosystémique (figure 4).

8. Devictor [2014] ou encore Thomas [2015] affirment que la notion de biodiversité représente déjà un glissement par rapport à la notion de nature, la première impliquant une classification, une organisation et finalement une quantification plus aisée, facilitant la mise en économie de la seconde. 
De la même manière, le nombre de citations dans la presse ne démontre pas de logique de substitution. En interrogeant la base bibliographique du journal Le Monde concernant les occurrences des termes " biodiversité », " service(s) écosystémique(s) 》 et " capital naturel ${ }^{9}$ ", deux observations s'imposent. La première est que les notions de services écosystémiques et de capital naturel sont très peu utilisées. La deuxième est que le nombre d'occurrences concernant le lexique proprement économique n'augmente pas - ou de façon insignifiante - depuis quinze ans, tandis qu'il croît fortement pour la biodiversité (figure 5).

Figure 4. Évolution de l'usage des notions de biodiversité et de service écosystémique dans la littérature scientifique

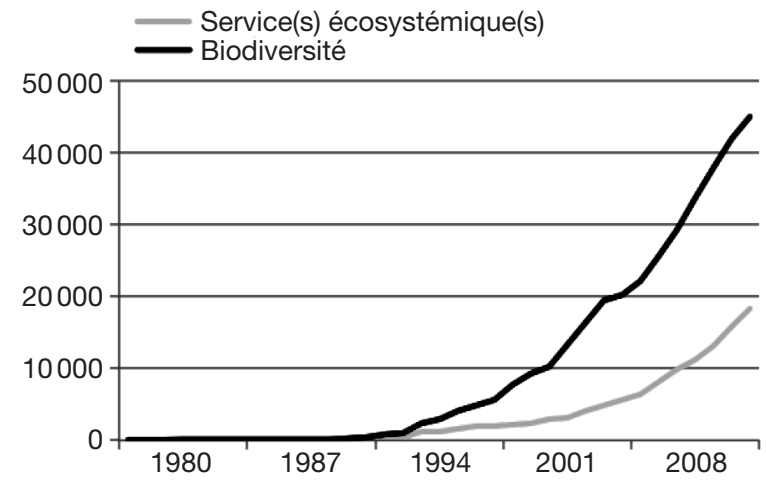

Source : SCOPUS, août 2016.

Figure 5. Nombre d'occurrences des termes "biodiversité ", " service écosystémique " et « capital naturel » dans Le Monde

Biodiversité

....... Service(s) écosystémique(s)

Capital naturel

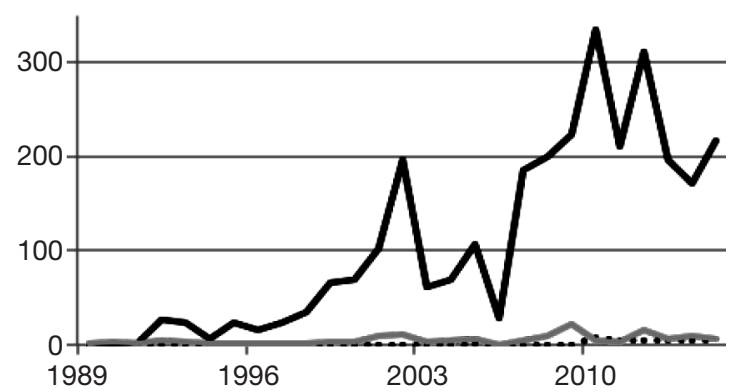

Source : Le Monde, août 2016.

9. Sur les enjeux entourant 1'usage de la notion de capital naturel, voir Åkerman [2003], Missemer [2018] et Nadal [2016]. 
Par ailleurs, lorsque la littérature mobilise les notions de services écosystémiques ou de capital naturel pour décrire la nature sous un angle économique, il semble que l'exercice se limite à un travail scientifique éloigné des sphères décisionnelles, contrairement à ce qui pourrait être imaginé (Laurans et al. [2013]). Et, dans les rares cas où ces évaluations sont mobilisées - comme par exemple dans la Directive-cadre sur l'eau pour évaluer la plus ou moins grande disproportion des coûts de restauration du bon état des eaux -, c'est avant tout pour offrir un outil de justification post-décisionnel bien différent d'une analyse coûts-bénéfices ex ante (Feuillette et al. [2016]).

Au regard des travaux récents, le succès relatif de la notion de service écosystémique, qui est à la croisée de concepts économiques et écologiques, s'explique probablement par la recherche d'un langage commun entre scientifiques venant de différentes disciplines et travaillant tous sur les interdépendances entre dynamiques écologiques et usages anthropiques (voir Roche et al. [2016]). La question de la mise en économie, qui apparaît en filigrane, n'est sans doute que secondaire par rapport à cette démarche heuristique. Globalement, le lexique issu de l'économie ne semble être qu'un langage parmi d'autres pour parler des espèces et milieux naturels.

\section{Évaluation monétaire ou équivalence écologique?}

L'origine de la monétarisation de la nature telle que l'entend la littérature contemporaine peut être fixée au moment de l'évaluation monétaire des bénéfices non marchands des dommages associés à la marée noire de l'Exxon Valdez en 1989 aux États-Unis (Milanesi [2011]). Pour comprendre ce qu'il s'est passé à ce moment-là, il faut revenir à la procédure NRDA (Natural Resource Damage Assessment) américaine, créée en 1986 par le Comprehensive Environmental Response, Compensation, and Liability Act (CERCLA), qui oblige les responsables de marées noires à mettre en œuvre une compensation pour les dommages environnementaux. Cette procédure a été reprise en 1990 par l'Oil Pollution Act (OPA). À l'origine, la procédure NRDA considérait que les pertes justifiant une compensation devaient être évaluées en valeur économique, ce qui validait une équivalence en somme d'utilités individuelles (Mazzotta, Opaluch et Grigalunas [1994]). Une difficulté concernait la manière de mesurer les valeurs de non-usage de la nature. Cette difficulté est devenue très prégnante au moment de la catastrophe de l'Exxon Valdez puisque la majeure partie des impacts concernait des zones où les populations humaines étaient absentes (Alaska). Dès lors, une solution proposée a été d'avoir recours à la méthode d'évaluation contingente qui permet d'estimer les valeurs de non-usage de la nature à partir de consentements à payer (Direction des études économiques et de l'évaluation environnementale [2005a], [2005b]; Desaigues et Point [1993]). Adoptée et mise en œuvre avec quelques précautions (Arrow et al. [1993]) dans le cadre de l'Exxon Valdez ${ }^{10}$, la méthode d'évaluation contingente a occupé le devant la scène académique, aux États-Unis comme en Europe, dans les années 1990 et 2000 (Levrel [2013]; Milanesi [2011]). Mais des difficultés méthodologiques

10. Sur les controverses liées à l'évaluation contingente dans le cadre précis de l'Exxon Valdez, voir Maas et Svorenčík [2017]. 
sont rapidement apparues pour évaluer les bénéfices perdus (voir Kahneman et Knetsch [1992] et Kahneman et Ritov [1994]), avec des controverses sur la capacité de ces outils à aider les cours de justice. Ces controverses, alimentées par de fortes incertitudes autour des évaluations réalisées, ont conduit les autorités américaines à se détourner de ces méthodes (Roach et Wade [2006]), comme en témoigne la jurisprudence autour de l'OPA (Thompson [2002]; Mazzotta, Opaluch et Grigalunas [1994], p. 158). Le législateur et les administrations ont alors abandonné le recours aux évaluations monétaires pour se tourner vers des évaluations biophysiques des composantes de la biodiversité. Exxon Valdez a en quelque sorte constitué un cas unique d'évaluation monétaire des valeurs de non-usage, non reproduit par la suite (Thompson [2002]; Zafonte et Hampton [2007]).

Aujourd'hui, les cours de justice n'insistent plus sur l'indemnisation du préjudice financier, mais sur la réparation du préjudice écologique, pour la nature elle-même. Cette réparation ne peut alors être réalisée qu'à partir d'actions biophysiques de restauration écologique. D'une équivalence économique on est ainsi passé à une équivalence écologique (Roach et Wade [2006]). Évidemment, la manière dont l'équivalence écologique peut être appréhendée donne lieu à des débats animés autour des unités d'équivalence, de la réalité même de l'équivalence, voire de la faisabilité de certaines compensations (Levrel, Pioch et Spieler [2012]; Levrel et al. [2015a]; Quétier et Lavorel [2011]). Mais nous ne sommes plus dans une évaluation économique de l'équivalence. En ce sens, nous assistons aujourd'hui davantage, dans ce domaine, à une démonétarisation des composantes de la biodiversité, plutôt qu'à une marche toujours plus rapide vers la monétarisation.

Certes, certains services peuvent donner lieu à une évaluation monétaire, notamment lorsqu'ils renvoient à des bénéfices concrets pour des activités et des acteurs précis : pollinisation, filtration de l'eau, etc. Mais de nombreux services sont hors marché et leur évaluation passe alors par des indicateurs biophysiques, sanitaires, d'accès, etc. (Cabral et al. [2016]; Levrel et al. [2014a]). Cette frontière à la monétarisation conduit à l'émergence d'évaluations multicritères des services écosystémiques (Goldstein et al. [2012]; Kareiva et al. [2011]; Levrel [2013]). Le pluralisme des indicateurs répond alors le plus souvent au pluralisme des valeurs portées par les individus, chacun insistant sur tel ou tel indicateur en fonction de son système de valeurs (Jacobs et al. [2016]).

Par ailleurs, l'approche par les coûts de maintien, qui consiste à évaluer les dépenses nécessaires pour maintenir le bon état de la biodiversité et des services écosystémiques dont elle est le support, recueille une adhésion croissante depuis plusieurs années (Bartelmus [2009]; Roach et Wade [2006]; Levrel et al. [2012]). C'est une méthode plus simple, qui s'inscrit dans le cadre d'une durabilité forte (Neumayer [1999]) où l'intégrité de la nature est défendue. Les normes environnementales générant des dépenses publiques et privées (pour améliorer la qualité de l'eau par exemple) deviennent le référentiel central pour établir des estimations monétaires fondées sur le coût de maintien et non plus sur la perte de valeur économique. L'économie retrouve alors sa place instrumentale, en fournissant un outil de comptabilité au service de politiques publiques qui restent autonomes dans la détermination de leurs objectifs. En France, une illustration récente de ce changement est offerte par l'évaluation des coûts de maintien du bon état écologique des eaux marines, dans le cadre de la Directivecadre Stratégie pour le milieu marin (DCSMM) (Levrel et al. [2014b]). 


\section{Retour des communs et hybridation des modes de régulation}

S'il semble exister des phénomènes de démonétarisation des services écosystémiques, les dynamiques de privatisation et de marchandisation de la nature doivent aussi être nuancées. L'exemple des paiements pour services environnementaux (PSE) et des banques de compensation pour les zones humides aux États-Unis, régies respectivement par le Wetland Reserve Program (WRP) et le Clean Water Act (CWA), mérite à cet égard un examen précis, dès lors que ces mécanismes sont parfois jugés comme typiques des processus contemporains de mise en économie de la biodiversité (Dauguet [2015]; Robertson [2004]; Spash [2011]; Walker et al. [2009]). En effet, il apparaît en réalité que ces outils ne conduisent pas à une privatisation ni à une marchandisation du vivant (Boisvert [2015a]; Hahn et al. [2015]).

Pour ce qui concerne la privatisation tout d'abord, les PSE et les banques de compensation dans le WRP et le CWA exigent l'adoption de servitudes environnementales (conservation easements) sur les terrains concernés (100\% des cas pour les banques de compensation et autour de $90 \%$ pour les PSE en zones humides). Cela conduit à transférer les droits privés d'usage sur les zones humides à des $\mathrm{ONG}$ ou à des agences publiques dont la mission est de protéger les écosystèmes au nom de l'intérêt général (Levrel et al. [2017]; Vaissière et Levrel [2015]). Les servitudes sont transférées au nouveau propriétaire en cas de vente du terrain, ce qui assure un respect des contraintes environnementales à long terme. Ce sont aujourd'hui près de 90000 hectares de zones humides qui sont partiellement déprivatisés chaque année aux États-Unis, dont 80000 à travers le WRP et 10000 à travers le CWA (Ferris et Siikamäki [2009]; Scemama et al. [2015]).

En France, il n'existe pas encore de servitudes environnementales ${ }^{11}$, mais on observe des formes de déprivatisation par d'autres vecteurs. Ainsi, la dynamique de déprise agricole, déjà ancienne (Lamaison [1988]), a conduit à l'abandon de certains terrains perçus comme dépourvus de valeur. Les héritiers n'en font aucun usage. Et en trois générations, le nombre de propriétaires potentiels, parfois dispersés loin des parcelles, peut atteindre plusieurs centaines d'individus. Les terrains deviennent difficiles à vendre (et à acheter) en raison de la nécessaire régularisation de dizaines de droits de propriété privés potentiels. Cela génère des coûts de transaction et des frais d'acquisition disproportionnés au regard de la valeur des terrains. Et cela conduit indirectement à des dynamiques de remise dans le domaine commun de milliers d'hectares d'écosystèmes redevenus naturels (même si l'on parle souvent de friches), en particulier dans les zones isolées comme les massifs montagneux ou les îles. Ce phénomène toucherait, en France, entre 90000 et 110000 ha/an de terres agricoles évoluant en friches, landes et forêts (Pointereau et Coulon [2009], p. 109).

L'exemple de la chasse, abordé historiquement dans une section précédente, fournit dans la période contemporaine d'autres exemples de sorties de l'économie. L'arrêt Chassagnou de 1999 reconnaît que, pour des raisons éthiques et

11. La loi sur la reconquête de la biodiversité du 8 août 2016 a ouvert la voie à de potentielles servitudes environnementales à travers les obligations réelles environnementales (ORE), contraignantes également pour les propriétaires futurs. Il est cependant trop tôt pour parler de réelle servitude environnementale compte tenu de la nouveauté de l'outil et de la durée contractuelle limitée des ORE. 
personnelles, un propriétaire foncier peut ainsi revendiquer un droit de non-chasse sur ses propriétés (Domas-Descos [2012]). Cette innovation conduit à limiter les droits des chasseurs sur les terrains privés, contrairement aux possibilités ouvertes par la loi Verdeille de 1964. Les parcelles concernées bénéficient finalement d'une forme de servitude environnementale de non-chasse, qui disparaîtra cependant au bout de six mois après la vente du terrain si le nouveau propriétaire n'a pas fait valoir le même droit. Par ailleurs, la multiplication des espèces protégées de tout usage anthropique, depuis une trentaine d'années, tend également à restreindre les droits de chasse, et donc l'ampleur des droits privés sur la faune.

En ce qui concerne les phénomènes de marchandisation, les États ont, depuis les années 1970, sorti du domaine marchand des pans entiers de la biodiversité menacée par les logiques commerciales. La Convention sur le commerce international des espèces de faune et de flore sauvages menacées d'extinction (CITES), adoptée en 1973, protège aujourd'hui 5000 espèces animales et 30000 espèces végétales en interdisant ou en encadrant précisément les usages auxquels elles peuvent donner lieu. Cela n'a pas permis l'éradication du braconnage, mais constitue néanmoins un dispositif légal de sortie de l'économie pour certaines composantes de la biodiversité.

En fin de compte, ces divers exemples suggèrent que beaucoup de modes de régulation contemporains conduisent à des processus de déprivatisation ou de démarchandisation de la nature. Fondamentalement, ces mécanismes constituent des formes organisationnelles hybrides : droits privés et publics s'articulent, avec parfois un retour des communs (Ostrom [1990]); PSE et banques de compensation confèrent à l'État, et pas seulement aux acteurs privés, un rôle décisif, en tant que régulateur mais aussi en tant qu'acheteur et vendeur (Boisvert, Méral et Froger [2013]; Vaissière et Levrel [2015]). Le plus souvent, les modes de régulation mis en place sont donc très éloignés des formes idéelles de la théorie économique qui consacreraient la primauté des acteurs privés décentralisés et du marché parfait dans la gestion de la nature ${ }^{12}$.

\section{CONCLUSION}

Le travail critique mené ici vient compléter les travaux existants qui cherchent à clarifier la diversité des formes de mise en économie de la nature (Castree [2003]; Gómez-Baggenthun et Ruiz-Pérez [2011]). Il vient compléter également les travaux qui mettent en exergue les résistances (institutionnelles, sociales, politiques) auxquelles se heurtent souvent les mécanismes de mise en économie (Boisvert [2016]; Gerber et Gerber [2017]; O’Neill [2007]). Nous avons tâché à la fois de faire le point sur l'état des débats (par une revue de la littérature) et d'offrir toute une série de contrepoints, à la fois historiques et contemporains, sur la réalité des processus envisagés.

Notre premier enseignement réside dans le constat d'une relative instabilité de la littérature sur ce que signifient, précisément, les processus de mise en économie. Quelques définitions clés surgissent, mais rares sont les travaux qui

12. Ce hiatus entre modes de gestion théoriques et réalités pratiques est également souligné par Fairhead, Leach et Scoones ([2012], p. 247) pour la question de l'accaparement des terres. 
prennent la peine de circonscrire rigoureusement leur objet d'étude. De ce manque de précision découle souvent l'affirmation de liens de causalité entre l'usage d'un lexique économique appliqué à la nature, la mise en équivalence, la privatisation puis la marchandisation et enfin la financiarisation de la nature. Pourtant, les innovations économiques associées à la conservation des milieux naturels et des composantes de la biodiversité revêtent une complexité qui tend à nuancer une lecture trop linéaire de ces modes de gestion de l'environnement. La chaîne causale de mise en économie de la nature, tout comme sa contemporanéité, se heurtent à des contre-exemples aussi bien anciens que récents.

Les $\mathrm{XVII}^{\mathrm{e}}-\mathrm{XVIII}{ }^{\mathrm{e}}$ siècles, ainsi que le tournant du $\mathrm{XX}^{\mathrm{e}}$ siècle, fournissent des exemples marquants de mise en économie dont les caractéristiques (présence d'acteurs privés, vision de la nature comme un ensemble d'actifs, etc.) sont très proches de ce qui semble observable aujourd'hui.

La période contemporaine est également riche en enseignements pour mesurer l'ampleur des phénomènes décrits. Contrairement à une opinion répandue, l'évaluation monétaire des dommages environnementaux n'a pas remporté la bataille des idées ni la bataille juridique, et l'appréhension des pollutions se fait aujourd'hui à travers des équivalences plus écologiques qu'économiques ${ }^{13}$. C'est avant tout le pragmatisme qui semble présider aux choix des régulateurs : les marchés de compensation pour la biodiversité sont apparus en substitut des systèmes de permis individuels, car ceux-ci étaient jugés trop coûteux pour les administrations et peu efficaces du point de vue des normes réglementaires à faire respecter (Vaissière et Levrel [2015]).

Nous ne contestons en aucune façon l'existence de phénomènes de mise en économie de la nature, ni ses excès comme la création de brevets sur le vivant (Bonneuil et Thomas [2009]) ou la mise en place de systèmes de concession sur les stocks de pêche et les forêts dans les pays du Sud (Karsenty et al. [2008]). Nous adhérons par ailleurs à l'hypothèse selon laquelle la période contemporaine semble avoir quelques spécificités, comme le règne du calcul d'optimisation ou l'individualisation extrême des responsabilités (Pestre [2016], p. 22). Enfin, l'essor du new public management dans la gestion de la biodiversité (plans de gestion dans les espaces protégés, audits, discours orientés clients, etc.) et le développement de «business verts » fondés sur l'écocertification sont sans doute à observer de près pour mesurer leur impact sur les objectifs environnementaux.

Ces différentes réalités ne doivent cependant pas conduire à parler de mise en économie totale de la nature, qui ne deviendrait alors qu'un actif générant des services écosystémiques échangeables sur des marchés, via des acteurs privés qui seraient les nouveaux pivots de la régulation environnementale. De tels jugements s'apparentent peut-être à des scénarios possibles pour l'avenir, mais à partir de conjectures fragiles et non sur la base d'évidences empiriques. Les expériences de démonétarisation, déprivatisation ou encore démarchandisation décrites dans les sections précédentes ne sont pas anecdotiques, et participent à une logique globale de «decommodification » (Gerber et Gerber [2017]) qui doit également appeler l'attention des observateurs. La mise en économie de la nature mérite donc d'être appréhendée avec prudence.

13. Ce mouvement vers une écologisation des mesures d'équivalence pourrait s'inscrire dans ce que Martuccelli ([2014], p. 382) qualifie de « régime écologique de réalité » aujourd'hui « en gestation "). 


\section{RÉFÉRENCES BIBLIOGRAPHIQUES}

Åkerman M. [2003], « What Does "Natural Capital” Do? The Role of Metaphor in Economic Understanding of the Environment », Environmental Values, 12 (4), p. 431-448.

Arrow K., Solow R., Portney P. R., Leamer E. E., Radner R. et Schuman H. [1993], « Report of the NOAA Panel on Contingent Valuation », Federal Register, 58 (10), p. 1-67.

BAKKeR K. [2007], « The Commons versus the Commodity: Alter-Globalization, AntiPrivatization and the Human Right to Water in the Global South », Antipode, 39 (3), p. $430-455$.

BALAN B. [1975], « Premières recherches sur l'origine et la formation du concept d'économie animale », Revue d'histoire des sciences, 28 (4), p. 289-326.

BanerJeE S. B. [2003], « Who Sustains Whose Development? Sustainable Development and the Reinvention of Nature ", Organization Studies, 24 (1), p. 143-180.

Bartelmus P. [2009], « The Cost of Natural Capital Consumption: Accounting for a Sustainable World Economy », Ecological Economics, 68 (6), p. 1850-1857.

Bentham J. [1789], An Introduction to the Principles of Morals and Legislation, Londres, T. Payne and Son.

Bergandi D. et Galangau-Quérat F. [2008], « Le développement durable. Les racines environnementalistes d'un paradigme », Aster, 46, p. 31-43.

BoIsvert V. [2015a], « La compensation écologique : marché ou marchandage ? », Revue internationale de droit économique, 29, p. 183-209.

BoIsvert V. [2015b], "Les services écosystémiques : un nouveau concept ? », dans F. Thomas et V. Boisvert (dir.), Le pouvoir de la biodiversité. Néolibéralisation de la nature dans les pays émergents, Paris/Versailles, IRD Éditions / Quæ, p. 215-229.

BoIsverT V. [2016], « Des limites de la mise en marché de l'environnement. Des services écosystémiques aux "banques de conservation" », Écologie \& Politique, 52, p. 63-79.

Boisvert V., Caron A. et Rodary E. [2004], « Privatiser pour conserver ? Petits arrangements de la nouvelle économie des ressources avec la réalité », Revue Tiers Monde, 177 (1), p. 61-83.

Boisvert V., Méral P. et Froger G. [2013], « MBIs for Ecosystem Services: Institutional Innovation or Renovation? », Society and Natural Resources, 26 (10), p. 1122-1136.

Bonneuil C. [2015], « Une nature liquide ? Les discours de la biodiversité dans le nouvel esprit du capitalisme », dans F. ThOmas et V. Boisvert (dir.), Le pouvoir de la biodiversité. Néolibéralisation de la nature dans les pays émergents, Paris/Versailles, IRD Éditions / Quæ, p. 193-213.

Bonneuil C. et Thomas F. [2009], Gènes, pouvoirs et profits. Recherche publique et régimes de production des savoirs de Mendel aux OGM, Versailles/Paris, Quæ/Éditions Charles Léopold Mayer.

Borras Jr. S. M., Hall R., Scoones I., White B. et Wolford W. [2011], « Towards a Better Understanding of Global Land Grabbing: An Editorial Introduction », The Journal of Peasant Studies, 38 (2), p. 209-216.

Boudia S. [2016], « Des instruments pour mettre en économie l'environnement. L'économicisation par approximation et occultation ", Écologie \& Politique, 52, p. 45-61.

Braat L. et Ten Brink P. (dir.) [2008], The Cost of Policy Inaction (COPI): The Case of Not Meeting the 2010 Biodiversity Target - Report to the European Commission, Wageningue/Bruxelles, Alterra / The Institute for European Environmental Policy.

Bruce A. A. [1909], « The Conservation of Our Natural Resources and of Our National Strength and Virility », University of Pennsylvania Law Review and American Law Register, 58 (3), p. 125-162.

Budds J. et McGranahan G. [2003], " Are the Debates on Water Privatization Missing the Point? Experiences from Africa, Asia and Latin America », Environment and Urbanization, 15 (2), p. 87-113.

Büscher B. [2010], "Derivative Nature: Interrogating the Value of Conservation in "Boundless Southern Africa" ", Third World Quarterly, 31 (2), p. 259-276. 
Cabral P., Feger C., Levrel H., Chabolle M. et Basque D. [2016], « Assessing the Impact of Land-Cover Changes on Ecosystem Services: A First Step Toward Integrative Planning in Bordeaux, France », Ecosystem Services, 22 (B), p. 318-327.

Castree N. [2003], « Commodifying What Nature? », Progress in Human Geography, 27 (3), p. 273-297.

CAstree N. [2006], « From Neoliberalism to Neoliberalisation: Consolations, Confusions, and Necessary Illusions », Environment and Planning A, 38 (1), p. 1-6.

Castree N. [2008a], « Neoliberalising Nature: The Logics of Deregulation and Reregulation », Environment and Planning A, 40 (1), p. 131-152.

Castree N. [2008b], « Neoliberalising Nature: Processes, Effects, and Evaluations », Environment and Planning A, 40 (1), p. 153-173.

Castree N. [2010], « Neoliberalism and the Biophysical Environment 2: Theorising the Neoliberalisation of Nature », Geography Compass, 4 (12), p. 1734-1746.

Castree N. et Henderson G. [2014], « The Capitalist Mode of Conservation, Neoliberalism and the Ecology of Value », New Proposals: Journal of Marxism and Interdisciplinary Inquiry, 7 (1), p. 16-37.

Charlton G. [1658], Exercitationes Physico-Anatomica de Economia Animali, Amsterdam, Apud Joannem Ravesteynium.

Chevassus-au-Louis B., Salles J.-M. et Pujol J.-L. (dir.). [2009], Approche économique de la biodiversité et des services liés aux écosystèmes, Paris, Centre d'analyse stratégique.

Costanza R., D’Arge R., De Groot R., Farber S., Grasso M., Hannon B., Limburg K., Naeem S., O’Neill R. V., Paruelo J., Raskin R. G., Sutton P. et Van den Belt M. [1997], «The Value of the World's Ecosystem Services and Natural Capital », Nature, 387 , p. $253-260$.

Dauguet B. [2015], « Biodiversity Offsetting as a Commodification Process: A French Case Study as a Concrete Example », Biological Conservation, 192, p. 533-540.

DeREX J.-M. [2001], «Pour une histoire des zones humides en France (XVII ${ }^{\mathrm{e}}-\mathrm{XIX}{ }^{\mathrm{e}}$ siècle) », Histoire \& Sociétés Rurales, 15, p. 11-36.

Derex J.-M. [2017], La mémoire des étangs et des marais, Paris, Ulmer.

Desaigues B. et Point P. [1993], Économie du patrimoine naturel, Paris, Economica.

Devictor V. [2014], « La protection de la nature : une double tension éthique et scientifique », Journal international de bioéthique, 25, p. 29-43.

Diderot D. et D'Alembert J. (dir.) [1755], Encyclopédie, ou Dictionnaire raisonné des sciences, des arts et des métiers, Paris, Briasson, David, Le Breton et Durand, t. V.

Direction des Études ÉCONOMiQues et DE L'ÉVALUation ENVIRONNEMENTALE [2005a], Valorisation des aménités liées aux usages récréatifs des rivières, Paris, Ministère de l'Écologie et du Développement Durable.

Direction DES ÉTUdes ÉCONOMiQues et De L'ÉVALUATION ENVIRONNEMENTALE [2005b], Guide technique pour la mise en cuvre de la méthode d'évaluation contingente, Paris, Ministère de l'Écologie et du Développement Durable.

Domas-Descos A. [2012], « Exercice du droit de chasse et droit de propriété », Économie rurale, 327-328, p. 114-125.

DufFy R. [2014], « Interactive Elephants: Nature, Tourism and Neoliberalism », Annals of Tourism Research, 44 (1), p. 88-101.

EhrLich P. R. et Mooney H. A. [1983], « Extinction, Substitution and Ecosystem Services », BioScience, 33 (4), p. 248-254.

Espeland W. N. et Stevens M. L. [1998], «Commensuration as a Social Process », Annual Review of Sociology, 24, p. 313-343.

Estève C. [2004], « Le droit de chasse en France de 1789 à 1914. Conflits d'usage et impasses juridiques », Histoire \& Sociétés Rurales, 21, p. 73-114.

Fairhead J., Leach M. et Scoones I. [2012], « Green Grabbing: A New Appropriation of Nature? », The Journal of Peasant Studies, 39 (2), p. 237-261.

Felli R. [2015], «Environment, Not Planning: The Neoliberal Depoliticisation of Environmental Policy by Means of Emissions Trading ", Environmental Politics, 24 (5), p. 641-660. 
FelLi R. [2016], La grande adaptation. Climat, capitalisme et catastrophe, Paris, Seuil.

FerRIS J. et SiIKAMÄKI J. [2009], « Conservation Reserve Program and Wetland Reserve Program: Primary Land Retirement Programs for Promoting Farmland Conservation », RFF Backgrounder, août.

Feuillette S., Levrel H., Boeuf B., Blanquart S., Gorin O., Monaco G., Penisson B. et Robichon S. [2016], « Methodological and Political Issues Raised by Cost-Benefit Analysis: The Case of the WFD Implementation in France ", Environmental Science and Policy, 57, p. 79-85.

FourCADE M. [2011], "Cents and Sensibility: Economic Valuation and the Nature of "Nature" ", American Journal of Sociology, 116 (6), p. 1221-1277.

Fox S. R. [1981], The American Conservation Movement: John Muir and His Legacy, Madison, University of Wisconsin Press.

Fressoz J.-B. [2012], L'Apocalypse joyeuse, Paris, Seuil.

Fressoz J.-B. [2013], « Payer pour polluer. L'industrie chimique et la compensation des dommages environnementaux, 1800-1850», Histoire \& Mesure, 28 (1), p. 145-186.

Gerber J.-D. et Gerber J.-F. [2017], « Decommodification as a Foundation for Ecological Economics », Ecological Economics, 131, p. 551-556.

Goldstein J. H., Caldarone G., Duarte T. K., Ennaanay D., Hannahs N., Mendoza G., Polasky S., Wolny S. et Daily G. C. [2012], « Integrating Ecosystem-Service Tradeoffs into Land-Use Decisions », Proceedings of the National Academy of Sciences, 109 (19), p. 7565-7570.

Gómez-Baggethun E., De Groot R. S., Lomas P. L. et Montes C. [2010], « The History of Ecosystem Services in Economic Theory and Practice: From Early Notions to Markets and Payment Schemes », Ecologial Economics, 69 (6), p. 1209-1218.

Gómez-Baggenthun E. et Ruiz-Pérez M. [2011], " Economic Valuation and the Commodification of Ecosystem Services », Progress in Physical Geography, 35 (5), p. 613-628.

GreEne S. [2004], « Indigenous People Incorporated? Culture as Politics, Culture as Property in Pharmaceutical Bioprospecting », Current Anthropology, 45 (2), p. 211-237.

HAECKEL E. [1874], Histoire de la création des êtres organisés d'après les lois naturelles, Paris, Reinwald.

Hahn T., McDermott C., Ituarte-Lima C., Schultz M., Green T. et Tuvendal M. [2015], «Purposes and Degrees of Commodification: Economic Instruments for Biodiversity and Ecosystem Services Need Not Rely on Markets or Monetary Valuation », Ecosystem Services, 16, p. 74-82.

HaYs S. P. [1959], Conservation and the Gospel of Efficiency, Pittsburgh, University of Pittsburgh Press.

JACOBS S. et al. [2016], «A New Valuation School: Integrating Diverse Values of Nature in Resource and Land Use Decisions », Ecosystem Services, 22 (B), p. 213-220.

Jerneck A., Olsson L., Ness B., Anderberg S., Baier M., Clark E., Hickler T., Hornborg A., Kronsell A., Lövbrand E. et Persson J. [2011], « Structuring Sustainability Science », Sustainability Science, 6 (1), p. 69-82.

Kahneman D. et Knetsch J. L. [1992], « Valuing Public Goods: The Purchase of Moral Satisfaction ", Journal of Environmental Economics and Management, 22 (1), p. 57-70.

Kahneman D. et Ritov I. [1994], « Determinants of Stated Willingness to Pay for Public Goods: A Study in the Headline Method », Journal of Risk and Uncertainty, 9 (1), p. 5-37.

Kareiva P., Tallis H., Ricketts T. H., Daily G. C. et Polasky S. [2011], Natural Capital: Theory and Practice of Mapping Ecosystem Services, Oxford, Oxford University Press.

Karsenty A., Drigo I. G., Piketty M. G. et Singer B. [2008], « Regulating Industrial Forest Concessions in Central Africa and South America », Forest Ecology and Management, 256 (7), p. 1498-1508.

Kellogg V. L. et Doane R. W. [1915], Elementary Textbook of Economic Zoology and Entomology, New York, Henry Holt \& Co.

Kosoy N. et Corbera E. [2010], « Payments for Ecosystem Services as Commodity Fetishism », Ecological Economics, 69 (6), p. 1228-1236. 
Kronenberg J. [2014], « What Can the Current Debate on Ecosystem Services Learn from the Past? Lessons from Economic Ornithology », Geoforum, 55, p. 164-177.

LAMAISON P. [1988], « De la transmission à l'abandon », Etudes rurales, 110-112, p. 9-27.

Laurans Y., Bouni C., Courtecuisse A., Dubien I. et Johannes B. [2001], « L'évaluation économique de la théorie à la pratique : l'espérience des SDAGE en France », Natures Sciences Sociétés, 9 (2), p. 17-28.

Laurans Y., Rankovic A., Billé R., Pirard R. et Mermet L. [2013], « Use of Ecosystem Services Economic Valuation for Decision Making: Questioning a Literature Blindspot », Journal of Environmental Management, 119, p. 208-219.

LÉVÊQuE C. [2007], «À propos de l'évaluation des écosystèmes pour le Millénaire : 1'économie de l'environnement à l'épreuve des faits », Natures Sciences Sociétés, 15 (1), p. $77-80$.

Levrel H. [2013], « La valeur de l'inaction et l'inaction de la valeur : quels indicateurs économiques pour travailler sur l'érosion de la biodiversité ? », dans M. BouAMrane, M. Antona, R. Barbault et M.-C. Cormier-Salem (dir.), Rendre possible : Jacques Weber, itinéraire d'un économiste passe-frontières, Versailles, Quæ, p. 241-259.

Levrel H., Cabral P., Marcone O. et Mongruel R. [2014a], «The Services Provided by Marine Ecosystems: Economic Assessments and Their Usages », dans A. MonAco et P. Prouzet (dir.), Value and Economy of Marine Resources, Hoboken (NJ), John Wiley \& Sons, p. 311-348.

Levrel H., Frascaria N., Hay J., Martin G. et Pioch S. (dir.). [2015a], Restaurer la nature pour atténuer les impacts du développement. Analyse des mesures compensatoires pour la biodiversité, Versailles, Quæ.

Levrel H., Hassan F., Scemama P. et Vaissière A.-C. [2015b], « Des innovations institutionnelles et organisationnelles pour accroître l'investissement dans le capital naturel», dans B. Perrissin Fabert (dir.), Nature et richesse des nations, Paris, Commissariat général au développement durable, p. 149-162.

Levrel H., Hay J., Bas A., Gastineau P. et Pioch S. [2012], « Coût d'opportunité vs coût du maintien des potentialités écologiques : deux indicateurs économiques pour mesurer le coûts de l'érosion de la biodiversité ", Natures Sciences Sociétés, 20 (1), p. 16-29.

Levrel H., Jacob C., Bailly D., Charles M., Guyader O., Aoubid S., Bas A., Cujus A., Frésard M., Girard S., Hay J., Laurans Y., Paillet J., Perez J. et Mongruel R. [2014b], « The Maintenance Costs of Marine Natural Capital: A Case Study from the Initial Assessment of the Marine Strategy Framework Directive in France », Marine Policy, 49, p. 37-47.

Levrel H., Pioch S. et Spieler R. [2012], « Compensatory Mitigation in Marine Ecosystems: Which Indicators for Assessing the "No Net Loss" Goal of Ecosystem Services and Ecological Functions? », Marine Policy, 36, p. 1202-1210.

Levrel H., Scemama P. et Vaissière A.-C. [2017], « Should We Be Wary of Mitigation Banking? Evidence Regarding the Risks Associated with this Wetland Offset Arrangement in Florida », Ecological Economics, 135, p. 136-149.

Liverman D. [2004], "Who Governs, at What Scale and at What Price? Geography, Environmental Governance, and the Commodification of Nature ", Annals of the Association of American Geographers, 94 (4), p. 734-738.

Luck G. W., Chan K. M. A., Eser U., Gómez-Baggethun E., Matzdorf B., Norton B. et Potschin M. B. [2012], "Ethical Considerations in On-Ground Applications of the Ecosystem Services Concept », BioScience, 62 (12), p. 1020-1029.

MaAs H. et Svorenčík A. [2017], " "Fraught with Controversy": Organizing Expertise Against Contingent Valuation », History of Political Economy, 49 (2), p. 315-345.

McAfEe K. [2010], « The Contradictory Logic of Global Ecosystem Services Markets », Development and Change, 43 (1), p. 105-131.

McAfee K. et Shapiro E. N. [2010], « Payments for Ecosystem Services in Mexico: Nature, Neoliberalism, Social Movements, and the State », Annals of the Association of American Geographers, 100 (3), p. 579-599.

McConnell G. [1954], « The Conservation Movement: Past and Present », The Western Political Quarterly, 7 (3), p. 463-478. 
MartucCelli D. [2014], Les sociétés et l'impossible. Les limites imaginaires de la réalité, Paris, Armand Colin.

Mazzotta M., Opaluch J. J. et Grigalunas T. A. [1994], « Natural Resource Damage Assessment: The Role of Resource Restoration », Natural Resources Journal, 34 (1), p. $153-178$.

Milanesi J. [2011], « Une histoire de la méthode d'évaluation contingente », Genèses, 84, p. 6-24

MiLl J. S. [1863], Utilitarianism, Londres, Parker, Son \& Bourn.

Missemer A. [2017], Les économistes et la fin des énergies fossiles (1865-1931), Paris, Classiques Garnier.

Missemer A. [2018], « Natural Capital as an Economic Concept: History and Contemporary Issues », Ecological Economics, 143, p. 90-96.

Nadal A. [2016], «The Natural Capital Metaphor and Economic Theory », Real-World Economics Review, 74, p. 64-84.

National Conservation Commission [1909], Report of the National Conservation Commission, Washington (D. C.), Government Printing Office, vol. I.

Nelson R. R. et Winter S. G. [1982], An Evolutionary Theory of Economic Change, Cambridge (Mass.), Harvard University Press.

Neumayer E. [1999], Weak versus Strong Sustainability: Exploring the Limits of Two Opposing Paradigms, Cheltenham, Edward Elgar.

O'Connor M. et Spash C. L. [1999], Valuation and the Environment: Theory, Methods and Practices, Cheltenham, Edward Elgar.

O'NeILl J. [2007], Markets, Deliberation and Environment, Londres, Routledge.

Ostrom E. [1990], Governing the Commons: The Evolution of Institutions for Collective Action, Cambridge, Cambridge University Press.

Palmer T. S. [1899], "A Review of Economic Ornithology in the United States ", dans Yearbook of the Department of Agriculture for 1899, Washington (D. C.), US Department of Agriculture, p. 259-292.

Pestre D. [2016], « La mise en économie de l'environnement comme règle. Entre théologie économique, pragmatisme et hégémonie politique », Écologie \& Politique, 52, p. 19-44.

Pinchot G. [1908], "The Foundations of Prosperity », The North American Review, 188 (636), p. 740-752.

Pinchot G. [1910], The Fight for Conservation, New York, Doubleday, Page \& Co.

Pinchot G. [1924], « Wages, Margins and Anthracite Prices », Annals of the American Academy of Political and Social Science, 111, p. 61-81.

Podolinsky S. [1880a], « Le socialisme et la théorie de Darwin », La Revue socialiste, 3, p. 129-148.

Podolinsky S. [1880b], " Le travail humain et la conservation de l'énergie », Revue internationale des sciences, 5, p. 57-70.

Pointereau P. et Coulon F. [2009], «Abandon et artificialisation des terres agricoles », Courrier de l'environnement de l'INRA, 57, p. 109-120.

Quesnay F. [1758], « Tableau économique, première version », rééd. dans C. Théré, L. Charles et J. Perrot (dir.), Euvres économiques complètes et autres textes de François Quesnay, Paris, INED, 2005, t. I, p. 397-403.

QuÉTIER F. et LAVOREL S. [2011], « Assessing Ecological Equilavence in Biodiversity Offset Schemes: Key Issues and Solutions », Biological Conservation, 144 (12), p. 2991-2999.

Rhoads S. N. [1898], " "Noxious" or "Beneficial”? False Premises in Economic Zoology », The American Naturalist, 32 (380), p. 571-581.

RoAch B. et WADE W. W. [2006], « Policy Evaluation of Natural Resource Injuries Using Habitat Equivalency Analysis », Ecological Economics, 58 (2), p. 421-433.

Robertson M. M. [2004], « The Neoliberalization of Ecosystem Services: Wetland Mitigation Banking and Problems in Environmental Governance », Geoforum, 35 (3), p. 361-373.

Robertson M. M. [2006], « The Nature that Capital Can See: Science, State, and Market in the Commodification of Ecosystem Services », Environment and Planning D, 24 (3), p. $367-387$ 
Robertson M. M. [2012], « Measurement and Alienation: Making a World of Ecosystem Services », Transactions of the Institute of British Geographers, 37 (3), p. 386-401. Trad. fr. : "Mesure et aliénation. Créer un monde de services écosystémiques », Écologie \& Politique, 52, 2016, p. 81-105.

Roche P., Geizzendorffer I., Levrel H. et Maris V. (dir.) [2016], Valeurs de la biodiversité et des services écosystémiques. Perspectives interdisciplinaires, Versailles, Quæ.

ROGER J. [1993], Les sciences de la vie dans la pensée française au XVIII siècle, Paris, Albin Michel.

Scemama P., Levrel H., Biotron R., Cabral P. et Vaissière A.-C. [2015], " Analyses spatio-temporelle du marché de la compensation des zones humides aux États-Unis », dans H. Levrel, N. Frascaria, J. Hay, G. Martin et S. Pioch (dir.), Restaurer la nature pour atténuer les impacts du développement. Analyse des mesures compensatoires pour la biodiversité, Versailles, Quæ, p. 102-115.

Schabas M. [2005], The Natural Origins of Economics, Chicago, The University of Chicago Press.

Schroter M., Van der Zanden E. H., Van Oudenhoven A. P. E., Remme R. P., SernaChavez H. M., De Groot R. S. et Opdam P. [2014], « Ecosystem Services as a Contested Concept: A Synthesis of Critique and Counter-Arguments », Conservation Letters, 7 (6), p. 514-523.

Spash C. L. [2011], « Terrible Economics, Ecosystems and Banking », Environmental Values, 20 (2), p. 141-145.

Sullivan S. [2013], « Banking Nature? The Spectacular Financialisation of Environmental Conservation », Antipode, 45 (1), p. 198-217.

Thomas F. [2015], « Introduction. Le pouvoir de la biodiversité », dans F. Thomas et V. BoIsvert (dir.), Le pouvoir de la biodiversité. Néolibéralisation de la nature dans les pays émergents, Paris/Versailles, IRD Éditions / Quæ, p. 9-40.

Thompson D. B. [2002], « Valuing the Environment: Courts' Struggles with Natural Resource Damages », Environmental Law, 32 (1), p. 57-89.

Thompson E. P. [1975], Whigs and Hunters: The Origin of the Black Act, Londres, Penguin Books.

Turnhout E., Waterton C., Neves K. et Buizer M. [2013], « Rethinking Biodiversity: From Goods and Services to "Living With" ", Conservation Letters, 6 (3), p. 154-161.

VAissiÈre A.-C. et Levrel H. [2015], « Biodiversity Offset Markets: What Are They Really? An Empirical Approach to Wetland Mitigation Banking », Ecological Economics, 110, p. 81-88.

Walker S., Brower A. L., Stephens R. T. T. et Lee W. G. [2009], « Why Bartering Biodiversity Fails », Conservation Letters, 2 (4), p. 149-157.

Wolloch N. [2016], Nature in the History of Economic Thought: How Natural Resources Became an Economic Concept, Londres, Routledge.

Zafonte M. et HAmpton S. [2007], « Exploring Welfare Implications of Resource Equivalency Analysis in Natural Resource Damage Assessments ", Ecological Economics, 61 (1), p. 134-145. 
ANNEXE

Tableau A1. Publications les plus citées dans le domaine de la mise en économie de la nature

\begin{tabular}{|c|c|c|c|c|}
\hline Titre & Auteur(s) & Journal & Année & $\begin{array}{l}\mathrm{N}^{\text {bre }} \text { de } \\
\text { citations }\end{array}$ \\
\hline $\begin{array}{l}\text { Neoliberalising Nature: The Logics } \\
\text { of Deregulation and Reregulation }\end{array}$ & Castree & $\begin{array}{l}\text { Environment and } \\
\text { Planning A }\end{array}$ & 2008 & 328 \\
\hline $\begin{array}{l}\text { The History of Ecosystem Services } \\
\text { in Economic Theory and Practice... }\end{array}$ & $\begin{array}{l}\text { Gómez- } \\
\text { Baggethun et al. }\end{array}$ & $\begin{array}{l}\text { Ecological } \\
\text { Economics }\end{array}$ & 2010 & 282 \\
\hline $\begin{array}{l}\text { Green Grabbing: A New } \\
\text { Appropriation of Nature? }\end{array}$ & $\begin{array}{l}\text { Fairhead, Leach } \\
\text { et Scoones }\end{array}$ & $\begin{array}{l}\text { Journal of Peasant } \\
\text { Studies }\end{array}$ & 2012 & 239 \\
\hline $\begin{array}{l}\text { Payments for Ecosystem Services } \\
\text { as Commodity Fetishism }\end{array}$ & $\begin{array}{l}\text { Kosoy et } \\
\text { Corbera }\end{array}$ & $\begin{array}{l}\text { Ecological } \\
\text { Economics }\end{array}$ & 2010 & 236 \\
\hline $\begin{array}{l}\text { Neoliberalizing Nature? Market } \\
\text { Environmentalism in Water... }\end{array}$ & Bakker & $\begin{array}{l}\text { Annals of the } \\
\text { Association } \\
\text { of American } \\
\text { Geographers }\end{array}$ & 2005 & 208 \\
\hline $\begin{array}{l}\text { Neoliberalising Nature: Processes, } \\
\text { Effects, and Evaluations }\end{array}$ & Castree & $\begin{array}{l}\text { Environment and } \\
\text { Planning A }\end{array}$ & 2008 & 195 \\
\hline $\begin{array}{l}\text { The Commons versus the } \\
\text { Commodity: Alter-Globalization... }\end{array}$ & Bakker & Antipode & 2007 & 186 \\
\hline $\begin{array}{l}\text { The Nature that Capital Can See: } \\
\text { Science, State, and Market... }\end{array}$ & Robertson & $\begin{array}{l}\text { Environment and } \\
\text { Planning D }\end{array}$ & 2006 & 154 \\
\hline Commodifying What Nature? & Castree & $\begin{array}{l}\text { Progress } \\
\text { in Human } \\
\text { Geography }\end{array}$ & 2003 & 143 \\
\hline $\begin{array}{l}\text { Who Sustains Whose Development? } \\
\text { Sustainable Development and... }\end{array}$ & Banerjee & $\begin{array}{l}\text { Organization } \\
\text { Studies }\end{array}$ & 2003 & 133 \\
\hline $\begin{array}{l}\text { Are the Debates on Water } \\
\text { Privatization Missing the Point? ... }\end{array}$ & $\begin{array}{l}\text { Budds et } \\
\text { McGranahan }\end{array}$ & $\begin{array}{l}\text { Environment and } \\
\text { Urbanization }\end{array}$ & 2003 & 125 \\
\hline $\begin{array}{l}\text { Payments for Ecosystem Services in } \\
\text { Mexico: Nature, Neoliberalism... }\end{array}$ & $\begin{array}{l}\text { McAfee et } \\
\text { Shapiro }\end{array}$ & $\begin{array}{l}\text { Annals of the } \\
\text { Association } \\
\text { of American } \\
\text { Geographers }\end{array}$ & 2010 & 116 \\
\hline $\begin{array}{l}\text { Economic Valuation and the } \\
\text { Commodification of Ecosystem... }\end{array}$ & $\begin{array}{l}\text { Gómez- } \\
\text { Baggethun et } \\
\text { Ruiz-Pérez }\end{array}$ & $\begin{array}{l}\text { Progress } \\
\text { in Physical } \\
\text { Georgraphy }\end{array}$ & 2011 & 115 \\
\hline $\begin{array}{l}\text { Who Governs, at What Scale and at } \\
\text { What Price? Geography... }\end{array}$ & Liverman & $\begin{array}{l}\text { Annals of the } \\
\text { Association } \\
\text { of American } \\
\text { Geographers }\end{array}$ & 2004 & 113 \\
\hline $\begin{array}{l}\text { Cost-Benefit Analysis and } \\
\text { Environmental Policy }\end{array}$ & Pearce & $\begin{array}{l}\text { Oxford Review of } \\
\text { Economic Policy }\end{array}$ & 1998 & 84 \\
\hline $\begin{array}{l}\text { The Contradictory Logic of Global } \\
\text { Ecosystem Services Markets }\end{array}$ & McAfee & $\begin{array}{l}\text { Development and } \\
\text { Change }\end{array}$ & 2012 & 82 \\
\hline
\end{tabular}


Tableau A2. Publications les plus citées dans le domaine de la mise en économie de la biodiversité

\begin{tabular}{|c|c|c|c|c|}
\hline Titre & Auteur(s) & Journal & Année & $\begin{array}{l}\mathrm{N}^{\text {bre }} \text { de } \\
\text { citations }\end{array}$ \\
\hline $\begin{array}{l}\text { Green Grabbing: A New } \\
\text { Appropriation of Nature? }\end{array}$ & $\begin{array}{l}\text { Fairhead, Leach } \\
\text { et Scoones }\end{array}$ & $\begin{array}{l}\text { Journal of Peasant } \\
\text { Studies }\end{array}$ & 2012 & 239 \\
\hline $\begin{array}{l}\text { Payments for Ecosystem Services } \\
\text { as Commodity Fetishism }\end{array}$ & $\begin{array}{l}\text { Kosoy et } \\
\text { Corbera }\end{array}$ & $\begin{array}{l}\text { Ecological } \\
\text { Economics }\end{array}$ & 2010 & 236 \\
\hline $\begin{array}{l}\text { The Nature that Capital Can See: } \\
\text { Science, State, and Market... }\end{array}$ & Robertson & $\begin{array}{l}\text { Environment and } \\
\text { Planning D }\end{array}$ & 2006 & 154 \\
\hline $\begin{array}{l}\text { Who Sustains Whose Development? } \\
\text { Sustainable Development and... }\end{array}$ & Banerjee & $\begin{array}{l}\text { Organization } \\
\text { Studies }\end{array}$ & 2003 & 133 \\
\hline $\begin{array}{l}\text { Payments for Ecosystem Services in } \\
\text { Mexico: Nature, Neoliberalism... }\end{array}$ & $\begin{array}{l}\text { McAfee et } \\
\text { Shapiro }\end{array}$ & $\begin{array}{l}\text { Annals of the } \\
\text { Association } \\
\text { of American } \\
\text { Geographers }\end{array}$ & 2010 & 116 \\
\hline $\begin{array}{l}\text { Economic Valuation and the } \\
\text { Commodification of Ecosystem... }\end{array}$ & $\begin{array}{l}\text { Gómez- } \\
\text { Baggethun et } \\
\text { Ruiz-Pérez }\end{array}$ & $\begin{array}{l}\text { Progress } \\
\text { in Physical } \\
\text { Georgraphy }\end{array}$ & 2011 & 115 \\
\hline $\begin{array}{l}\text { The Contradictory Logic of Global } \\
\text { Ecosystem Services Markets }\end{array}$ & McAfee & $\begin{array}{l}\text { Development and } \\
\text { Change }\end{array}$ & 2012 & 82 \\
\hline Structuring Sustainability Science & Jerneck et al. & $\begin{array}{l}\text { Sustainability } \\
\text { Science }\end{array}$ & 2011 & 80 \\
\hline $\begin{array}{l}\text { Measurement and Alienation: } \\
\text { Making a World of Ecosystem... }\end{array}$ & Robertson & $\begin{array}{l}\text { Transactions } \\
\text { of the Institute } \\
\text { of British } \\
\text { Geographers }\end{array}$ & 2012 & 62 \\
\hline $\begin{array}{l}\text { Banking Nature? The Spectacular } \\
\text { Financialisation of... }\end{array}$ & Sullivan & Antipode & 2013 & 61 \\
\hline $\begin{array}{l}\text { Indigenous People Incorporated? } \\
\text { Culture as Politics, Culture... }\end{array}$ & Greene & $\begin{array}{l}\text { Current } \\
\text { Anthropology }\end{array}$ & 2004 & 61 \\
\hline $\begin{array}{l}\text { Ecosystem Services as a Contested } \\
\text { Concept: A Synthesis of... }\end{array}$ & Schroter et al. & $\begin{array}{l}\text { Conservation } \\
\text { Letters }\end{array}$ & 2014 & 51 \\
\hline $\begin{array}{l}\text { Ethical Considerations in } \\
\text { On-Ground Applications of the } \\
\text { Ecosystem... }\end{array}$ & Luck et al. & Bioscience & 2012 & 50 \\
\hline $\begin{array}{l}\text { Rethinking Biodiversity: From } \\
\text { Goods and Services to... }\end{array}$ & Turnhout et al. & $\begin{array}{l}\text { Conservation } \\
\text { Letters }\end{array}$ & 2013 & 48 \\
\hline
\end{tabular}

